\title{
Analysis of Nonlinear Dynamic Structures
}

\section{Hailay Kiros}

Department of Mechanical Engineering, Ethiopian Institute of Technology-Mekelle (EiT-M), P. O. Box: 3013, Mekelle University, Mekelle, Ethiopia (hailaykk@gmail.com, hailaykiros@yahoo.com)

\begin{abstract}
It is probable that all practical engineering structures are nonlinear to some extent. In this paper work a two degrees of freedom nonlinear system with zero memory was simulated, modeled and analyzed to show their behavior for different conditions. The limit of chaos was also determined. To achieve these results simulation of dynamic systems was used and analyzed using MATLAB. This was done using sweeping sine forces as an input and controlling the amplitude specially when determining the limits of chaos. Using harmonic balance and FRF good results were achieved and verified.
\end{abstract}

Keywords: Simulation, Nonlinear, Zero Memory, Chaos, MATLAB.

$\begin{array}{ll}\text { Notations } & \\ \mathrm{B} & \text { System Impedance Matrix } \\ \mathrm{c} & \text { Damping Coefficient } \\ \mathrm{F} & \text { Frequency Domain Force } \\ \mathrm{F} & \text { Fourier Transform } \\ \mathrm{f} & \text { Force } \\ \mathrm{G} & \text { Spectral Density } \\ \mathrm{L} & \text { Laplace Transform } \\ \mathrm{H} & \text { Frequency Response Function } \\ \mathrm{k} & \text { Spring Constant } \\ \mathrm{L} & \text { Length } \\ \mathrm{M} & \text { Constant Integer } \\ \mathrm{m} & \text { Mass } \\ \mathrm{N} & \text { Constant Integer } \\ \mathrm{n} & \text { Noise } \\ \mathrm{p} & \text { Arbitrary Constant } \\ \mathrm{t} & \text { Time } \\ \mathrm{w} & \text { Frequency in cycles/second } \\ \mathrm{X} & \text { Frequency Domain Displacement } \\ x & \text { Displacement } \\ \dot{x} & \text { Velocity } \\ \ddot{x} & \text { Acceleration } \\ \mathrm{Y} & \text { Frequency Domain Output } \\ y & \text { Output } \\ z & \text { Input } \\ \beta, \alpha & \text { Constants } \\ \phi & \text { Angle } \\ \gamma^{2} & \text { Coherence } \\ & \end{array}$




\section{Indices}

m Multiple

F Input Force

P, q Reference Points

$\mathrm{X} \quad$ Response

$1,2,3, \mathrm{M} \quad$ Integers

\section{Abbreviations}

$\begin{array}{ll}\text { FRF } & \text { Frequency Response Function } \\ \text { MIMO } & \text { Multiple Input Multiple Output } \\ \text { MISO } & \text { Multiple Output Single Input } \\ \text { rms } & \text { Root Mean Square }\end{array}$

\section{INTRODUCTION}

Structures can be categorized as linear and nonlinear according to their reaction to an input force. Many engineering structures are treated as linear despite it is probable that all practical engineering structures are nonlinear to some extent. The nonlinearity can be caused by one or a combination of several factors. Mathematically the so called principle of superposition is used in most cases to differentiate linear systems from the nonlinear which is defined as follows.

Assuming a system with some initial conditions responds to an input $z_{1}(t)$ with an output $y_{1}(t)$ and to an input $z_{2}(t)$ with an output $y_{2}(t)$, then superposition holds if and only if the input $\alpha z_{1}(t)+\beta z_{2}(t)$ gives $\alpha y_{1}(t)+\beta y_{2}(t)$ as an output for all $\alpha$ and $\beta$. Systems which satisfy this condition are said to be linear.

The aim of this paper work is to be able to simulate, model and analyze nonlinear structures with zero memory in order to show their behavior for different conditions. It will also show a way in determining the limit of chaos in structures based on simulations. Hence two degrees of freedom nonlinear system was studied as lumped mass system and the study focuses on analysis of the nonlinearity behavior as well as determination of limit of chaos with respect to amplitude of the input force. This is done with the help of simulations which was done using MATLAB.

The most common types of nonlinearity encountered in dynamic testing are those due to polynomial stiffness and damping, clearance, impacts, friction and saturation effect (Worden and Tomlinson, 2001). In this work only the nonlinearity due to polynomial stiffness is considered. 


\section{METHODOLOGY}

In this work MATLAB simulations based on two types of nonlinear FRF construction methods are used for the analysis of the nonlinear system. These are Harmonic Balance and Multiple Input Multiple Output (MIMO) or Multiple Input Single Output (MISO) techniques.

\subsection{FRF of Nonlinear Systems}

FRF is the most widely used method in structural dynamics which gives information about the input-output properties of structures. The FRF gives most of the information necessary to specify the dynamics of a structure. Among these are resonances, anti-resonances, mode shapes, and phase. It can also be used to identify whether the system is linear or nonlinear. In the case of a linear system, the response to a sinusoid is always a sinusoid at the same frequency and FRF summarizes input/output process and does not depend on the amplitude of excitation. In the case of nonlinear systems, sinusoidal input results in response components at frequencies other than the excitation frequency and the FRF depends on the amplitude of excitation.

\subsection{Harmonic Balance}

The method of harmonic balance is one of the basic analytical techniques for approximating response of nonlinear system. To show how this approximate solution works, here the derivation is presented below (Worden and Tomlinson, 2001; Jaumouille et al., 2010).

Considering a single degree of freedom (SDOF) system with cubic non linearity as follows:

$$
m \ddot{x}+c \dot{x}+k x+k_{3} x^{3}=y(t)
$$

With the assumption that the response to sinusoidal excitation is a sinusoid at the same frequency and,

$$
\begin{aligned}
& x=X \sin (w t) \\
& m \ddot{x}+c \dot{x}+k x+k_{3} x^{3}=Y \sin (w t-\phi)
\end{aligned}
$$

Neglecting the higher harmonics, this yields

$$
-m w^{2} X \sin (w t)+c w X \cos (w t)+k X \sin (w t)+k_{3} X^{3} \sin ^{3}(w t) \quad=Y \sin (w t-\phi)
$$

After little elementary trigonometry this becomes

$$
\begin{aligned}
& -m w^{2} X \sin (w t)+c w X \cos (w t)+k X \sin (w t)+\quad k_{3} X^{3}(3 / 4 \sin (w t)-1 / 4 \sin (3 w t)) \\
& =Y \sin (w t) \cos \phi-Y \cos (w t) \sin \phi) \quad \ldots \ldots \ldots \ldots \ldots \ldots \ldots \ldots \ldots \ldots \ldots \ldots \ldots \ldots \ldots \ldots \ldots \ldots \ldots \ldots \ldots \ldots \ldots \ldots \ldots \ldots \ldots \ldots \ldots
\end{aligned}
$$

Equating for the same coefficients of both sides of (2.5) gives:

$$
\left(-m w^{2} X+k X+3 / 4 k_{3} X^{3}\right)=Y \cos \phi
$$




$$
c w X=-Y \sin \phi
$$

Squaring, adding and rearranging these equations yields

$$
\left|\frac{X}{Y}\right|=\frac{1}{\left(\left(-m w^{2}+k+\frac{3}{4} k_{3} X^{2}\right)^{2}+c^{2} w^{2}\right)^{\frac{1}{2}}}
$$

And the phase is obtained from the ratio of equations (2.6) and (2.7) as,

$$
\phi=\tan ^{-1} \frac{-c w}{-m w^{2}+k+\frac{3}{4} k_{3} X^{2}}
$$

\subsection{MIMO Identification Technique}

In order to show the MIMO identification technique for nonlinear systems, it is useful to start with linear system first in brief (Magnevall et al., 2006; Josefsson et al., 2007). So considering the linear system shown in figure 1 below, with $\mathrm{N}$ inputs and $\mathrm{M}$ outputs, and noise on the measured outputs, the $\mathrm{H}_{1}$ estimator can be expressed as:

$$
H_{1}=G_{X F} \cdot G_{F F}^{-1}
$$

Where $G_{X F}$ - is the cross-spectral density matrix with size $(M \times N), G_{F F}$ the auto-spectral density matrix with size $(M \times N)$ and $\mathrm{H}_{1}$ is the estimated transfer matrix with size $(M \times N)$.

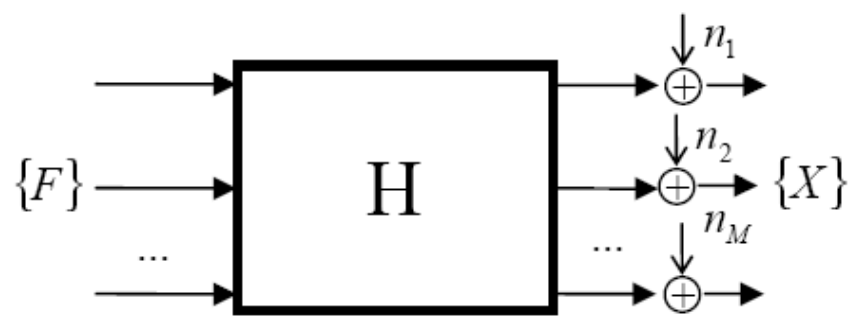

Figure 1. MIMO model with noise at the output.

Having this, the coherence function which is used as check of signal quality or linearity can be defined for single input single output $p$ and $q$ respectively as follows.

$$
\gamma_{p q}^{2}=\frac{\left|G_{X_{p} F_{q}}\right|^{2}}{G_{F_{q} F_{q}} \cdot G_{X_{p} X_{p}}}
$$

This ordinary coherence will give a value of unity if linear relationship exists for the single input and single output case. But for the case of MIMO/MISO, the coherence can show much less than unity, due to the influence of other inputs while there is perfect linear relationship between 
all inputs and outputs. Due to this reason a multiple coherence, which is shown below, is used for the MIMO case (Magnevall et al., 2006; Josefsson et al., 2007).

$$
\gamma_{m}^{2}=\frac{G_{X F} \cdot G_{F F}^{-1} \cdot G_{X F}^{H}}{G_{X X}}
$$

Where, $G_{X X}$ is auto-spectral density and $G_{X X}^{H}$ is its complex conjugate transpose.

Using MIMO technique MATLAB toolbox has been used for nonlinear systems (Ahlin and Lango, 2007). It is important to give brief explanation of the technique for the sake of clarity. Considering the Duffing's equation:

$$
m \cdot \ddot{x}+c \cdot \dot{x}+k \cdot x+p \cdot x^{3}=f
$$

Taking equation (2.13) into the frequency domain

$$
B \cdot X+p \cdot \mathscr{F}\left(x^{3}\right)=F
$$

Where, $B$ is the impedance of linear system and $\mathscr{F}($.$) is the Fourier transform. As is explained in$ (Magnevall et al., 2006) equation (2.14) is solved using the MIMO technique by the so called reverse path method as shown in the figure 2 below (Josefsson et al., 2007).

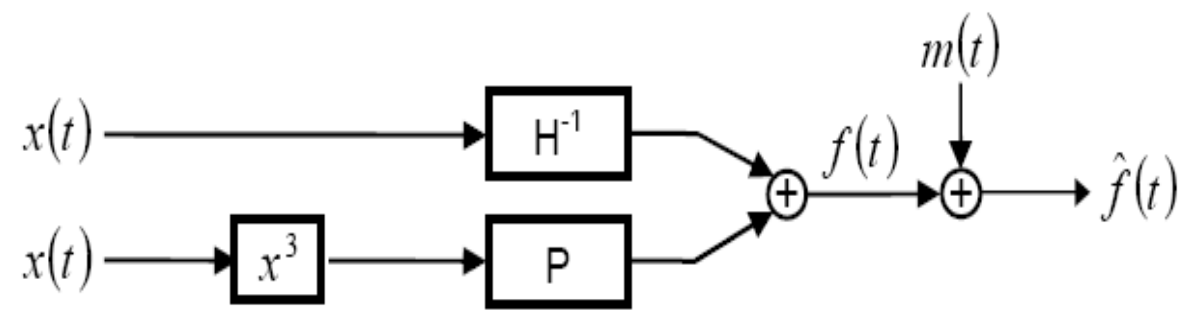

Figure 2. Reverse path method for solving Duffing's equation.

\section{ANALYSIS OF NONLINEAR LUMPED MASS SYSTEM}

A two degree of freedom system with cubic spring of zero memory attached between one of the masses and the ground is used to study a nonlinear system as shown in figure 3.

The equations of motion for this system can be written as follows.

$$
\begin{aligned}
& m_{1} \ddot{x}_{1}+c_{1}\left(\dot{x}_{1}-\dot{x}_{2}\right)+k_{1}\left(x_{1}-x_{2}\right)+p x_{1}^{3}=f(t) \\
& m_{2} \ddot{x}_{2}+c_{2} \dot{x}_{2}-c_{1}\left(\dot{x}_{1}-\dot{x}_{2}\right)+k_{2} x_{2}-k_{1}\left(x_{1}-x_{2}\right)=0
\end{aligned}
$$

Rearranging the above equations in a matrix form;

$\left[\begin{array}{cc}m_{1} & 0 \\ 0 & m_{2}\end{array}\right] \cdot\left\{\begin{array}{l}\ddot{x}_{1} \\ \ddot{x}_{2}\end{array}\right\}+\left[\begin{array}{cc}c_{1} & -c_{1} \\ -c_{1} & c_{1}+c_{2}\end{array}\right] \cdot\left\{\begin{array}{l}\dot{x}_{1} \\ \dot{x}_{2}\end{array}\right\}+\left[\begin{array}{cc}k_{1} & -k_{1} \\ -k_{1} & k_{1}+k_{2}\end{array}\right] \cdot\left\{\begin{array}{l}x_{1} \\ x_{2}\end{array}\right\}=\left\{\begin{array}{c}f(t)-p x_{1}^{3} \\ 0\end{array}\right\}$ 


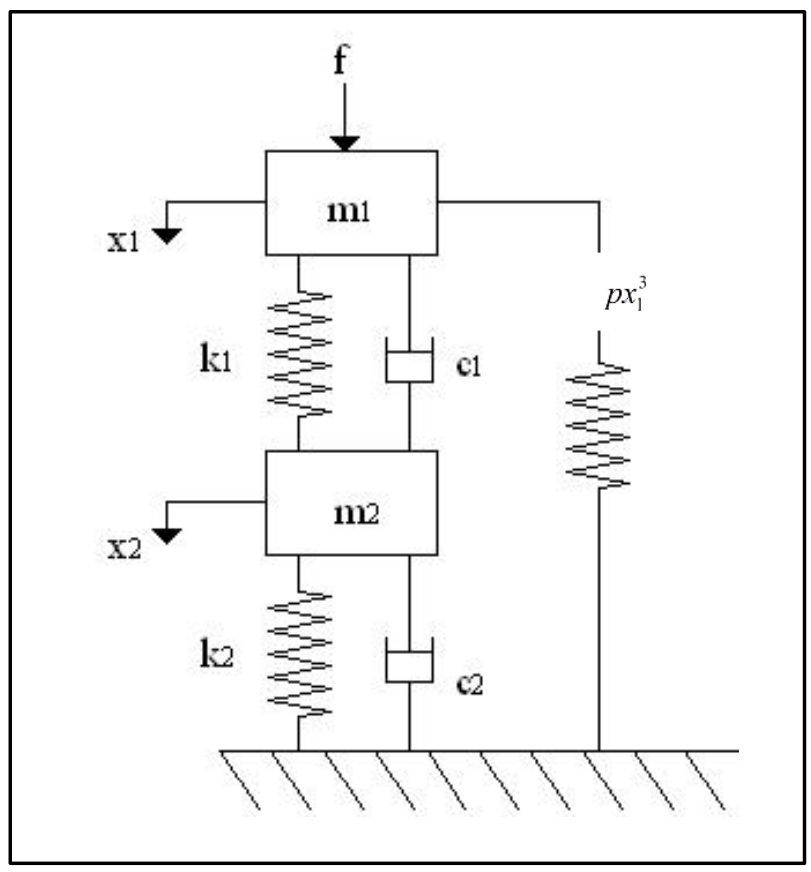

Figure 3. Two degrees of Freedom nonlinear lumped-mass system model.

Taking equation (3.3) into the frequency domain (Laplace Transform)

$\left[\left[\begin{array}{cc}m_{1} & 0 \\ 0 & m_{2}\end{array}\right] s^{2}+\left[\begin{array}{cc}c_{1} & -c_{1} \\ -c_{1} & c_{1}+c_{2}\end{array}\right] s+\left[\begin{array}{cc}k_{1} & -k_{1} \\ -k_{1} & k_{1}+k_{2}\end{array}\right]\right]\left\{\begin{array}{l}X_{1} \\ X_{2}\end{array}\right\}=\mathrm{L}\left\{\begin{array}{c}f(t)-p x_{1}^{3} \\ 0\end{array}\right\}$

This can be written as

$$
[B(s)]\{X\}+p \cdot \mathscr{L}\left(x_{1}^{3}\right)=F(s)
$$

Where $[B(s)]=\left[\begin{array}{cc}m_{1} & 0 \\ 0 & m_{2}\end{array}\right] s^{2}+\left[\begin{array}{cc}c_{1} & -c_{1} \\ -c_{1} & c_{1}+c_{2}\end{array}\right] s+\left[\begin{array}{cc}k_{1} & -k_{1} \\ -k_{1} & k_{1}+k_{2}\end{array}\right]$, is system impedance matrix and; $\mathscr{L}($.$) is Laplace transform.$

$$
\{X\}=\left\{\begin{array}{l}
X_{1} \\
X_{2}
\end{array}\right\} \text {, is displacement vector in the frequency domain }
$$

$F(s)$ is the Laplace transform of force $f(t)$.

Now equation (3.5) is the same as equation (2.14) and can be solved using the MIMO technique explained above.

A forward sine sweep force (Fig. 4) is applied to the system in figure 3 and analyzed using MATLAB (2005). This system acts as linear for very small values of the input force and starts to 
be nonlinear as the amplitude is increased. Figure 5(a) shows that the system is in the verge of linear and nonlinear with $0.2 \mathrm{~N}$ root mean square (rms) value of the input force. For a force of 2 $\mathrm{N}$ rms, the nonlinearity can be clearly seen for the first degree of freedom as shown in figure $5(b)$.

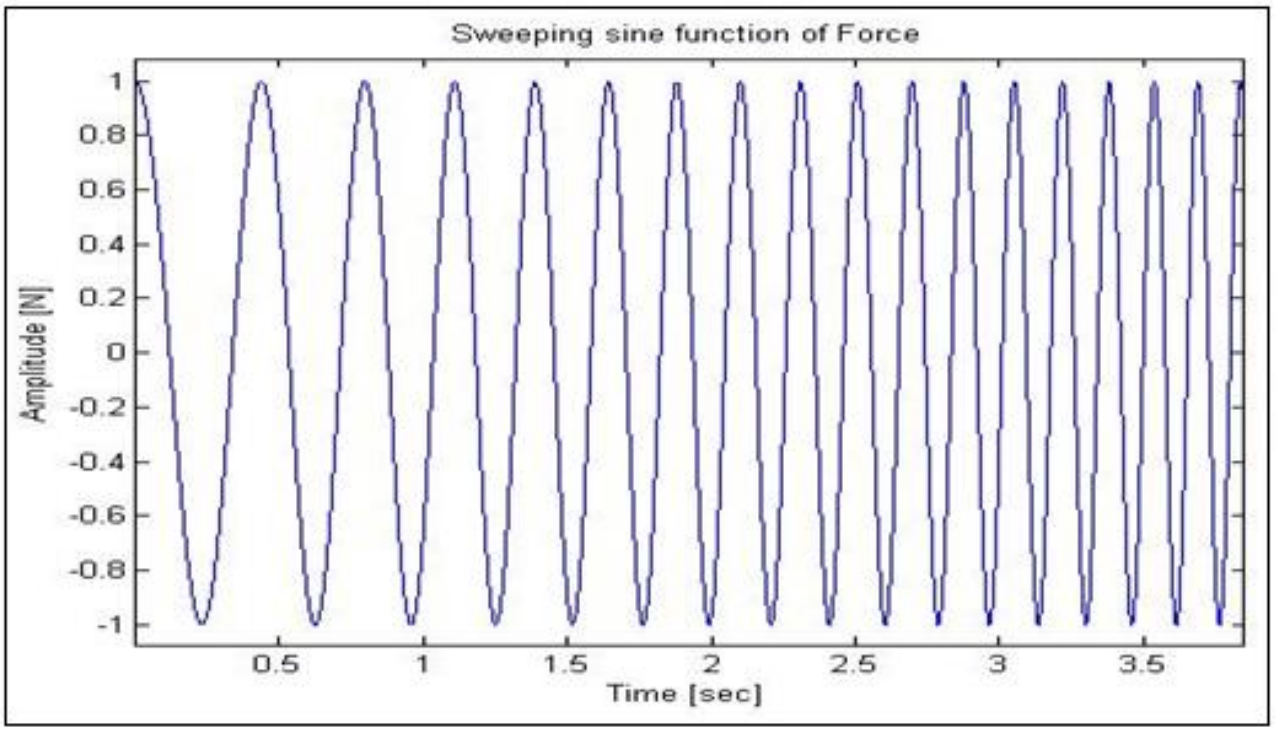

Figure 4. Example of sweeping sine function.
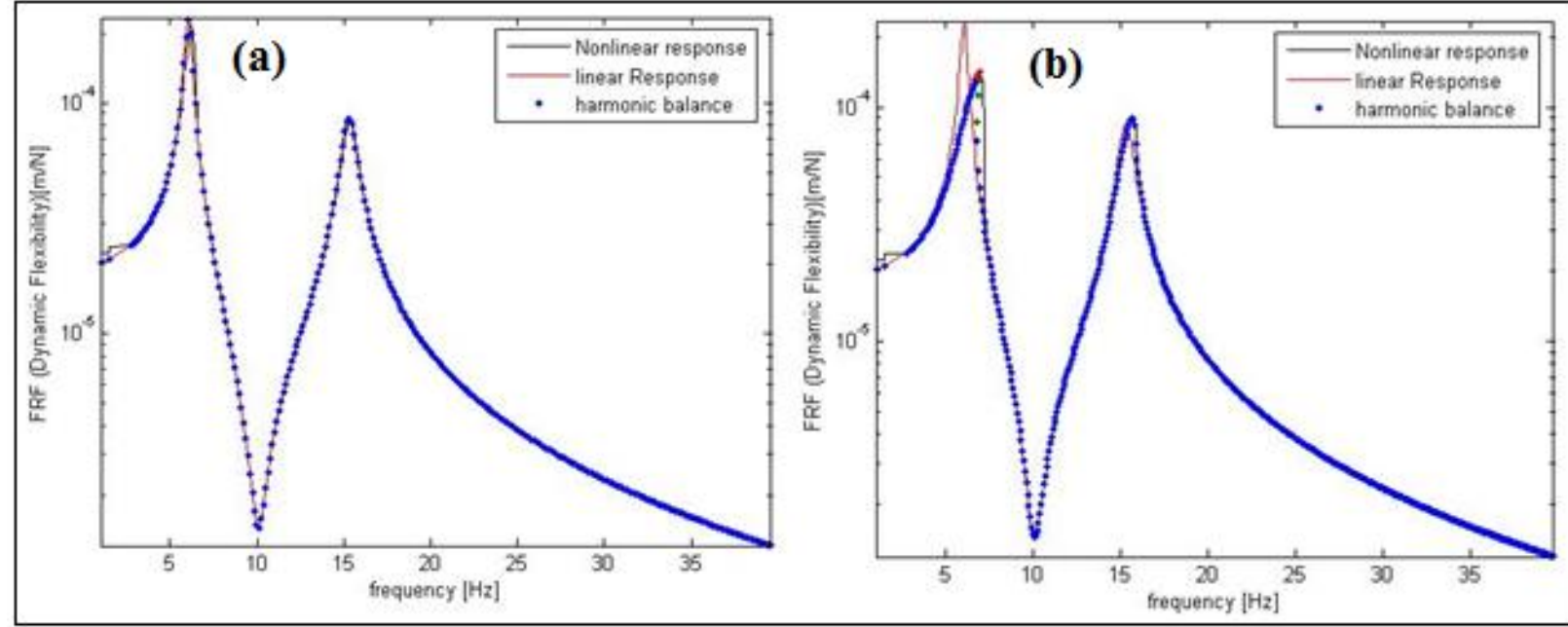

Figure 5. FRF of the two degree of freedom lumped mass system (a): linear response of the nonlinear structure at low amplitude of the input force $\mathrm{F}=0.2 \mathrm{~N}$ rms. (b): Starting of nonlinear response at amplitude of the input force $\mathrm{F}=2 \mathrm{~N} \mathrm{rms}$.

When the rms value of the input force is increased both resonances show nonlinearity as can be seen from figure 6(a) and (b). There is a jump of the response as compared to the harmonic 
balance for both resonances. This is due to the cubic nonlinearity which is introduced in the system. The harmonic balance is also showing that there are possibilities of getting three roots at one frequency as shown for the regions of green and red colors or around the tip of the curved part of the graphs which is the case for nonlinear conditions. In figure 6(b), around the second resonance, it is shown that the nonlinearity is stronger or more that the energy content was distributed to broader frequency range. In the same figure near the first resonance, there is some chaotic behavior shown.
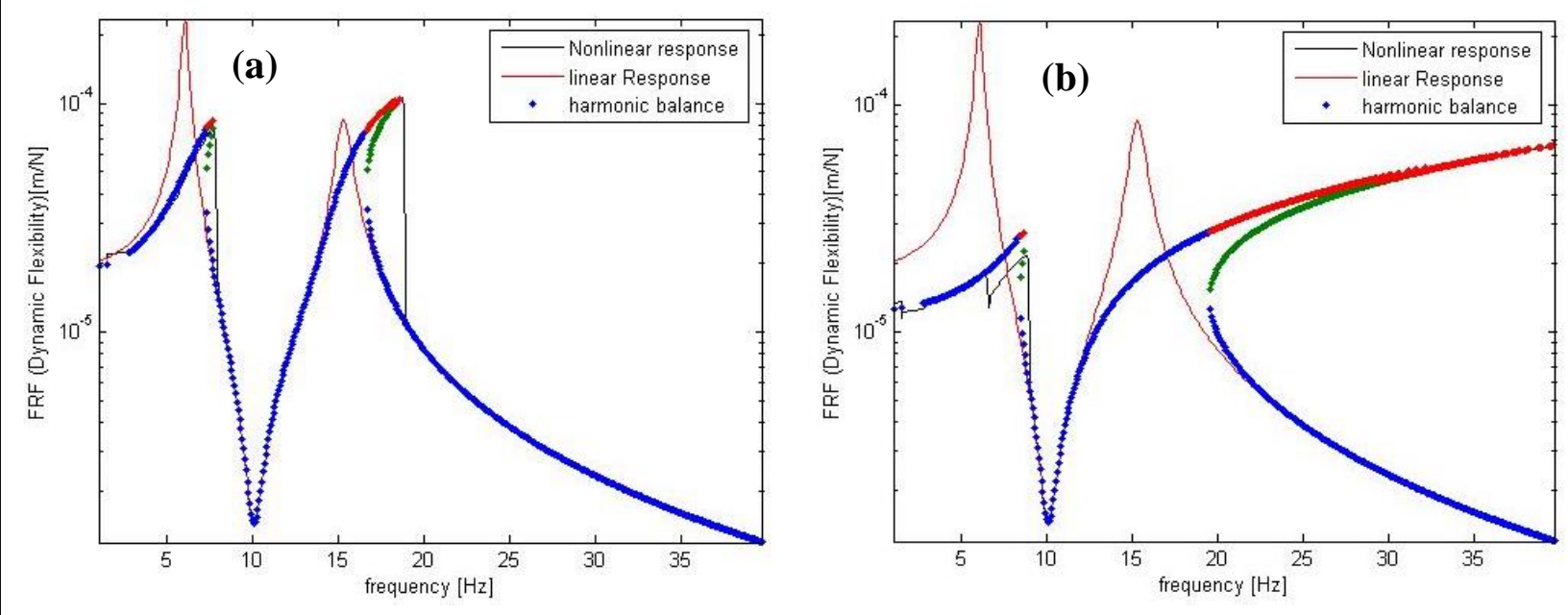

Figure 6(a) Nonlinear FRF of two degrees of freedom lumped mass system for $\mathrm{F}=5 \mathrm{~N}$ rms. Nonlinearity is shown around both the resonances; (b) Nonlinear FRF of two degrees of freedom lumped mass systems for $\mathrm{F}=25 \mathrm{~N}$ rms. Some chaotic behavior is shown around the first resonance and strong nonlinearity for the second resonance.

Nonlinear systems may get in to chaotic behavior when the amplitude of the input force is increased to some extent. The chaotic behavior of the lumped mass system is introduced in this section in order to show the possibility of determination for limit of chaos for multi degrees of freedom system considering the frequency region of interest in general. Hence as can be seen from figures 7 and 8 below, for an rms value of the input force of around $42 \mathrm{~N}$, the system shows chaos at both resonances. The frequency region of the chaos can be also known from the simulation as shown in Figures below. From figure 7, the first resonance shows chaotic behavior at around $7-10 \mathrm{~Hz}$ while the second resonance is around $12-16 \mathrm{~Hz}$. This is evident from figure 3.6, which is the waterfall diagram of the same response, as well. It is also evident that the chaotic behavior is more around the second resonance frequency. 


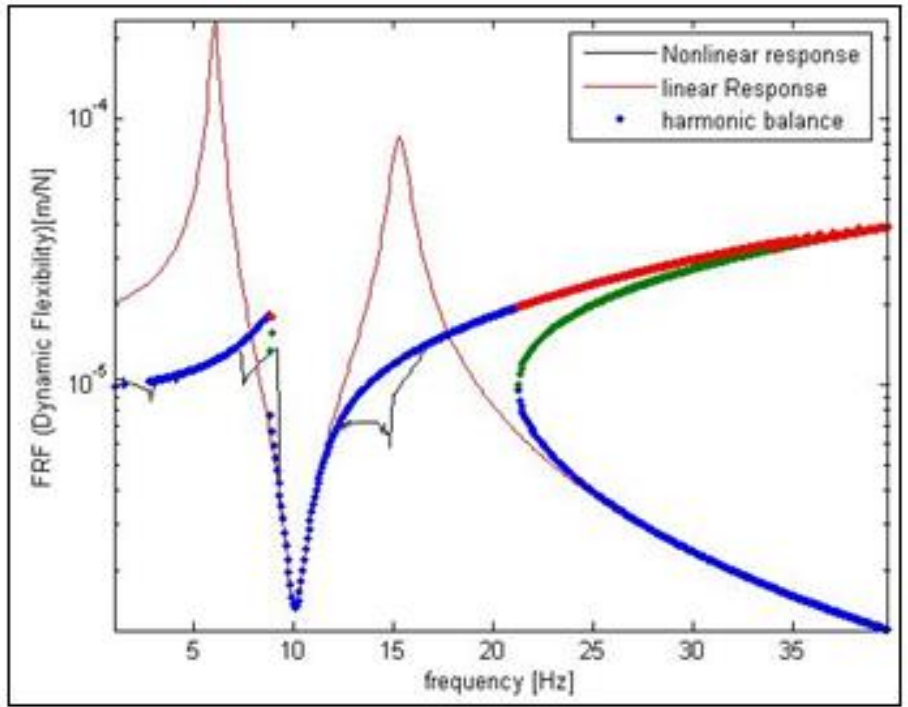

Figure 7. Chaotic FRF of the lumped mass system for $\mathrm{F}=42 \mathrm{~N}$ rms. Chaos shown at 7-10 $\mathrm{Hz}$ of the first resonance and $12-16 \mathrm{~Hz}$ of the second resonances.

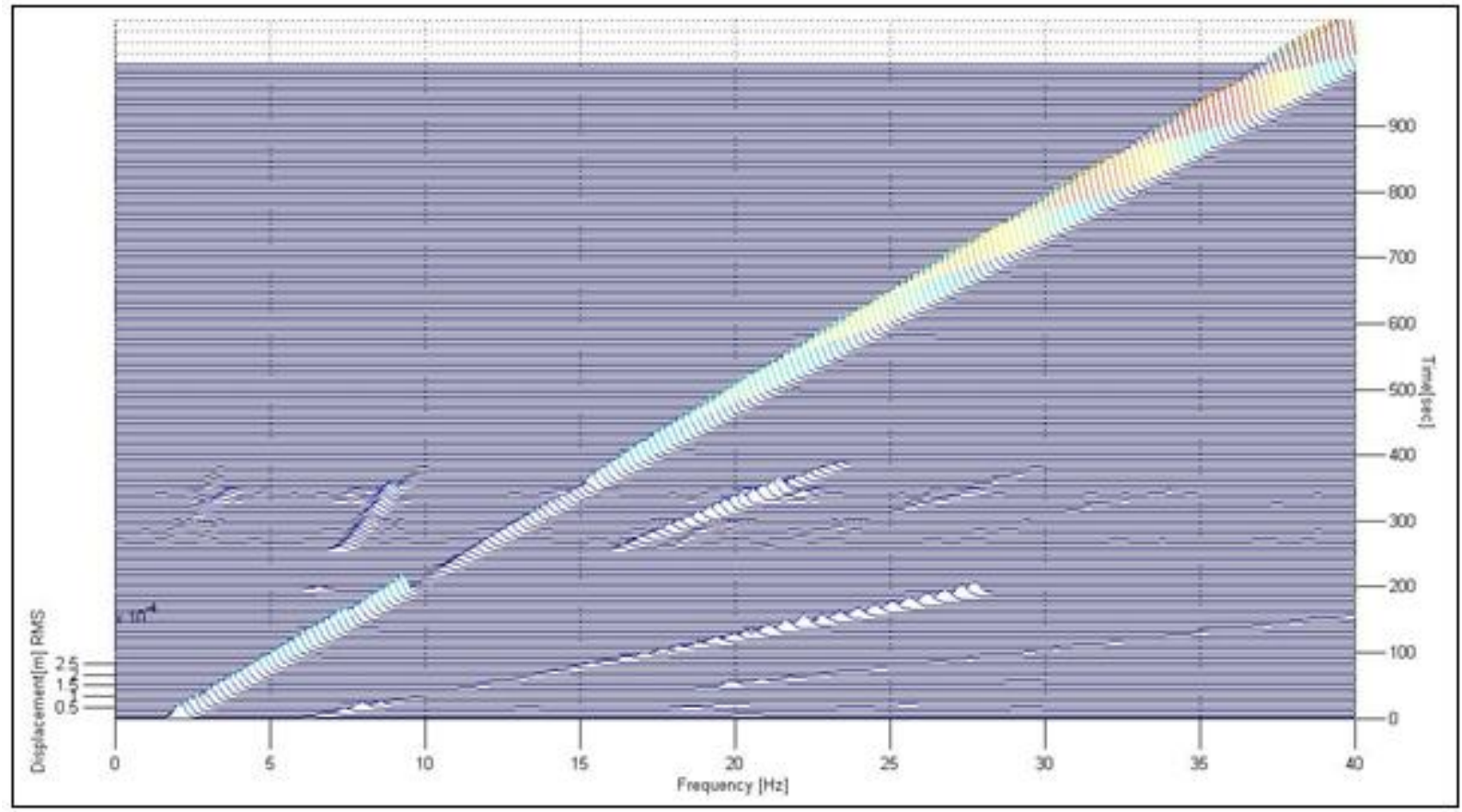

Figure 8. Chaotic Waterfall diagram of the lumped mass system's response for $\mathrm{F}=42 \mathrm{~N}$ rms.

This result shows that it is possible to determine the limit of chaos for two degrees of freedom system. This indicates also that the determination of limit of chaos for nonlinear systems is possible considering the first two resonances. 


\section{CONCLUSION}

Nonlinear systems with zero memory have been studied and analyzed based on the conventional MIMO technique and using MATLAB toolbox. Good results have been achieved for two degrees of freedom lumped-mass system.

Simulation was done for sweeping sine as an input force and used as a check for the existence of nonlinearity as well as chaos in the systems/structure. The limit of chaos was also determined using simulation in relation to the applied amplitude to the system. This is important in predetermining the limits for uncontrolled region for systems with the existed situation so that it will be in the safe region behaviorally.

In addition to this, as can be seen from figures 5 to 7, the system's linear parameters of nonlinear structure can be extracted using the reverse path method using some experimental tests of nonlinear structures. This is useful for the real structural systems which are nonlinear in nature and need to know parameters of their dynamic behavior like the damping ratio and stiffness and based on such kinds of parameters, modifications can easily be applied in order to avoid nonlinear behaviors in systems.

All the above simulation works and results can also be implemented for systems which are in real applications that can exhibit unexpected dynamic behaviors in order to take all necessary corrective actions, and to avoid risks with help of some kind experimental works which will help in getting the necessary data for the analysis.

\section{ACKNOWLEDGEMENT}

This work was carried out at the Department of Mechanical Engineering, Blekinge Institute of Technology, Karlskrona, Sweden. I would like to express my sincere appreciation to Professor Kjell Ahlin for his guidance and professional engagement and assistance throughout this work.

\section{REFERENCE}

Ahlin, K \& Lango, B. 2007. Toolbox for Simulations and Parameter identification of Nonlinear Mechanical Systems, IMAC XXV, Orlando.

Jaumouille, V., Sinou, J. J \& Petitjean, B. 2010. An Adaptive Harmonic Balance Method for predicting the non-linear dynamic responses of mechanical systems - Application to bolted structures. Journal of sound and vibration, 329(19): 4048-4067. 
Josefsson, A., Magnevall, M \& Ahlin, K. 2007. On Nonlinear Parameter Estimation with Random Noise Signals, IMAC XXV, Orlando.

Magnevall, M. Josefsson, A \& Ahlin, K. 2006. On Nonlinear Parameter Estimation, ISMA 2006, Leuven.

MATLAB. 2005. Computing Software v 7.0 (R14). The Mathworks, Inc.

Worden, K \& Tomlinson, G.R. 2001. Nonlinearity in Structural Dynamics, Institute of Physics Publishing, UK. 